\title{
Laparoscopic versus open hepatectomy for hepatocellular carcinoma with cirrhosis: a single-center propensity score matching analysis
}

\author{
Yongfei He ${ }^{1 \#}$, Dalang Fang ${ }^{2 \#}$, Tianyi Liang ${ }^{1}$, Shutian Mo ${ }^{1}$, Yuhua Meng ${ }^{3}$, Zijun Chen ${ }^{1}$, Shuqi Zhao ${ }^{1}$, \\ Yuan Liao ${ }^{1}$, Ketuan Huang ${ }^{1}$, Shunqiang Nong ${ }^{4}$, Weijie Zhou ${ }^{4}$, Chuangye Han ${ }^{1,5}$, Tao Peng ${ }^{1,5}$ \\ ${ }^{1}$ Department of Hepatobiliary Surgery, The First Affiliated Hospital of Guangxi Medical University, Nanning, China; ${ }^{2}$ Department of Breast \\ and Thyroid Surgery, The Affiliated Hospital of Youjiang Medical University for Nationalities, Baise, China; ${ }^{3}$ Department of Pathology, Shunde \\ Hospital, Southern Medical University (The First People's Hospital of Shunde, Foshan), Foshan, China; ${ }^{4}$ Clinical Laboratory, Baise People's \\ Hospital, Baise, China; ${ }^{5}$ Guangxi Key Laboratory of Enhanced Recovery after Surgery for Gastrointestinal Cancer, Nanning, China \\ Contributions: (I) Conception and design: T Peng, C Han; (II) Administrative support: T Peng; (III) Provision of study materials or patients: Y He, \\ T Liang; (IV) Collection and assembly of data: S Mo, Z Chen, S Zhao, Y Liao, K Huang; (V) Data analysis and interpretation: D Fang, Y Meng, S \\ Nong, W Zhou; (VI) Manuscript writing: All authors; (VII) Final approval of manuscript: All authors. \\ \#These authors contributed equally to this work. \\ Correspondence to: Tao Peng; Chuangye Han. Department of Hepatobiliary Surgery, The First Affiliated Hospital of Guangxi Medical University, \\ Nanning, China. Email: pengtaogmu@163.com; 383171884@qq.com.
}

Background: Laparoscopic hepatectomy $(\mathrm{LH})$ for hepatocellular carcinoma (HCC) remains controversial due to limited research. This study analyzed the oncology prognosis of patients who received LH treatment for HCC compared with conventional open hepatectomy $(\mathrm{OH})$.

Methods: We conducted a retrospective analysis of patients with cirrhosis who underwent hepatectomy for HCC between 2012 and 2018. Patients were divided into LH and OH groups, and the oncology outcomes were compared before and after 1:1 propensity score matching (PSM).

Results: A total of 403 patients with HCC cirrhosis who received LH $(n=112)$ and OH $(n=291)$ were enrolled. After PSM, 106 pairs of patients were matched. Compared with OH before and after PSM, there was no significant difference in overall survival (OS) and relapse-free survival (RFS) between the two groups. Tumor stage, Child-Pugh classification, venous tumor thrombus, tumor size $\geq 5 \mathrm{~cm}$, and microvascular invasion (MVI) were independent risk factors for postoperative OS in HCC patients with cirrhosis. Tumor size $\geq 5 \mathrm{~cm}$ and MVI were independent risk factors for RFS.

Conclusions: Patients with HCC who underwent LH had a similar OS and RFS compared with those who received traditional open surgery. Therefore, $\mathrm{LH}$ can be used as a safe and feasible treatment for patients with HCC.

Keywords: Hepatocellular carcinoma (HCC); laparoscopic hepatectomy (LH); open hepatectomy (OH); prognosis

Submitted Oct 15, 2021. Accepted for publication Nov 23, 2021.

doi: $10.21037 / \mathrm{atm}-21-5833$

View this article at: https://dx.doi.org/10.21037/atm-21-5833

\section{Introduction}

Hepatocellular carcinoma (HCC) is the third leading cause of cancer deaths worldwide. Approximately 50\% of new liver cancer patients are from China, making
HCC the second most common malignant tumor in China (1-3). Currently, treatment for HCC is divided into surgical treatment and non-surgical treatment, and surgical treatment includes tumor resection and liver 
transplantation. Due to the shortage of liver sources and the long waiting time for transplantation, liver transplantation cannot be performed clinically on a large scale. Hence, liver resection remains the primary surgical option for a potential cure. Unfortunately, most HCC patients already have cirrhosis by the time of diagnosis. HCC patients with cirrhosis have a higher risk of intraoperative bleeding, postoperative refractory ascites, and liver failure following tumor resection (4). Therefore, the technique requires a high level of surgical skill and experience.

Reich et al. completed the world's first laparoscopic hepatectomy (LH) in 1991, ushering in a new era of laparoscopic liver resections (5). As a relatively less invasive surgical method, this modified technique has attracted worldwide attention (6). LH has been reported to result in less blood loss, lower rates of postoperative complications, and shorter hospital stays than conventional open hepatectomy $(\mathrm{OH})(7)$. However, most surgeons have expressed concerns about tumor spread in the endoscopy environment, difficulty locating tumor boundaries, and difficulty controlling bleeding when tumors are close to important blood vessels, resulting in the slow promotion and uptake of LH amongst surgeons worldwide. With the popularization of technology and the improvement in endoscopic surgical techniques, the theoretical and technical aspects of LH have become well established, and the feasibility, safety, and effectiveness of LH have been gradually confirmed.

Although significant progress has been made in $\mathrm{LH}$ technology, its application in HCC patients remains controversial, with concerns remaining about tumor spread and adverse oncology outcomes (8). In 2018, Ramirez et al. compared open abdominal surgery with laparoscopic or robot-assisted minimally invasive surgery for patients with early cervical cancer and found that minimally invasive resection had no advantage in the treatment prognosis of early cervical cancer but had a higher recurrence rate and increased risk of death (9). The findings have also sparked a fierce debate in the field of hepatobiliary surgery. A study of 2,804 patients undergoing $\mathrm{LH}$ showed no significant difference in perioperative complication rates and mortality between LH and OH (10). For HCC patients with cirrhosis, laparoscopic surgery has also demonstrated good short- and long-term outcomes $(11,12)$. However, most of these reports involve small liver resections, and the issue remains controversial.

Our center is located in a region with a high incidence of
HCC in China, and the number of hepatectomy performed is increasing year by year. Therefore, this study can help local doctors choose surgical procedures and provide theoretical reference for other doctors. The purpose of this study was to analyze the survival results of patients with HCC complicated with cirrhosis treated with $\mathrm{LH}$ and $\mathrm{OH}$ in our center. To reduce the potential selection bias inherent in retrospective studies, we used a propensity score matching (PSM) analysis to determine the differences in survival outcomes between the two surgical procedures. We present the following article in accordance with the STROBE reporting checklist (available at https://dx.doi. org/10.21037/atm-21-5833).

\section{Methods}

\section{Study subjects}

We retrospectively collected data from patients with HCC, without obvious surgical contraindications, who underwent hepatectomy and complicated with cirrhosis from 2012 to 2018 in the First Affiliated Hospital of Guangxi Medical University. A total of 403 patients with HCC complicated with cirrhosis were enrolled and formed the basis of this study. Since our study focused on LH, based on the number of LH cases, after 1:1 PSM, there were 106 patients in the open group and 106 patients in the laparoscopic group (Figure 1). Baseline, clinical, and pathological data and survival outcome data were collected for retrospective analysis.

\section{Inclusion and exclusion criteria}

This study is a retrospective study. In order to unify terminology and facilitate understanding, so the diagnosis of primary liver cancer is based on The Guidelines for the Diagnosis and Treatment of Primary Liver Cancer in China (2019 Edition) (13). The patient inclusion criteria were as follows: (I) male or female patients aged 18-75 years; (II) a diagnosis of HCC with cirrhosis confirmed by postoperative pathology; (III) a preoperative evaluation confirming the feasibility of HCC resection. The exclusion criteria included (I) secondary HCC, (II) early HCC intervention (e.g., transcatheter arterial embolization, radiofrequency ablation, or portal vein embolization), (III) HCC rupture and hemorrhage, (IV) postoperative follow-up loss, (V) non-radical resection, and (VI) a follow-up time less than 6 months. 


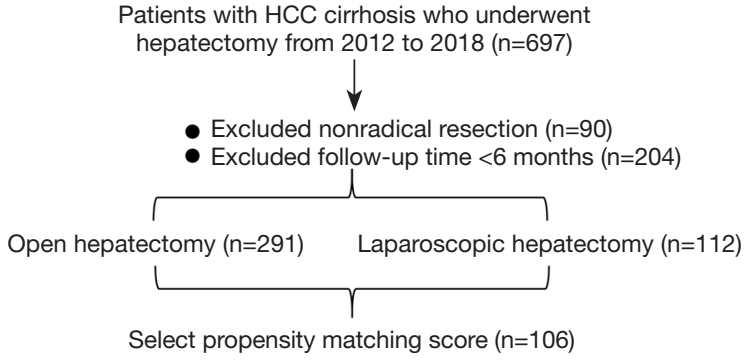

Figure 1 The screening flow chart of HCC patients with cirrhosis. HCC, hepatocellular carcinoma.

\section{Data collection}

Data on 403 cases were obtained through our inpatient and outpatient information management systems and were based on the inclusion and exclusion criteria. The available data included sex, age, hepatitis background, smoking and alcohol intake (alcohol intake $>30 \mathrm{~g} /$ day for men and $>20 \mathrm{~g} /$ day for women), tumor assessment (presence of portal vein cancer thrombus, tumor size, and number), intraoperative conditions (surgical method, operative time, blood loss, blood transfusion volume, etc.), pathology [degree of differentiation, cirrhosis, microvascular invasion (MVI), etc.], postoperative complications, and length of hospital stay. Postoperative complications were recorded during hospitalization at the same surgery or within 30 days after discharge and were assessed according to the Clavien-Dindo classification system (14). Patients or family members were followed up through our information management system and by telephone, with OS defined as the time from surgery to death or the date of the last follow-up. RFS was defined as the time from surgery to the date of a diagnosis of $\mathrm{HCC}$ recurrence.

\section{Statistical analysis}

Continuous variables are presented as mean (standard deviation) and independent sample $t$-test is used. The categorical variables are presented in terms of numbers (percentage), using the Chi-square test or Fisher's exact probability method as required. Kaplan-Meier method was used to calculate the cumulative mortality and recurrence rates between the open and endoscopic groups, and logrank method was used to compare the differences in mortality and recurrence rates between the two groups. In this study, propensity score matching was used to eliminate confounding effects of included variables. We matched patients in the open and endoscopic groups at a ratio of $1: 1$, and factors related to overall survival (OS) and relapsefree survival (RFS) were included in the propensity scoring model, including age, sex, body mass index (BMI), smoking, alcohol, $\alpha$-fetoprotein (AFP), diabetes mellitus (DM), hypertension, Barcelona clinic liver cancer stage (BCLC), China liver cancer stage (CNLC), Child-Pugh grade, liver fluke, tumor size and number, hepatitis virus infection, pathological stage, MVI, operation time, and blood loss. we used propensity score for non-replace-matching, and matched parameters were $($ Method $=$ Nearlyst, CLIper $=0.2 * \mathrm{SD})$. COX proportional risk model was used for univariate and multivariate analysis to identify risk factors associated with OS and RFS. $\mathrm{P}<0.05$ was considered statistically significant. All analyses were performed using $\mathrm{R}$ software (https://www.r-project.org/).

\section{Ethical statement}

The study was conducted in accordance with the Declaration of Helsinki (as revised in 2013). This study was approved by the Ethics Committee of the First Affiliated Hospital of Guangxi Medical University (No. 2021-KY-E277). Individual consent for this retrospective analysis was waived.

\section{Results}

\section{Baseline characteristics}

We conducted a retrospective analysis of 403 HCC patients with cirrhosis who underwent hepatectomy from 2012 to 2018. There were 291 cases $(72.2 \%)$ in the laparotomy group and 112 cases $(27.8 \%)$ in the laparoscopy group. Baseline data before PSM showed that CNLC stage $(\mathrm{P}=0.021)$, venous tumor thrombus $(\mathrm{P}=0.027)$, hepatitis ( $\mathrm{P}=0.022)$, duration of surgery $(\mathrm{P}=0.013)$, bleeding $(\mathrm{P}=0.04)$, and length of hospitalization $(\mathrm{P}<0.001)$ were significantly different between the groups (Table 1). After 1:1 PSM, 106 patients remained in each group, and all clinicopathological features were well balanced between the two groups (Table 2, Figure 2).

\section{Univariate and multivariate analyses of OS and RFS predictions}

Before PSM, univariate analysis showed that BCLC stage $\mathrm{B}-\mathrm{C}(\mathrm{P}=0.008)$, CNLC stage III $(\mathrm{P}=0.009)$, venous tumor 


\section{Page 4 of 15}

He et al. Analysis of LH and OH of HCC with liver cirrhosis

Table 1 Patients' baseline characteristics before propensity score matching

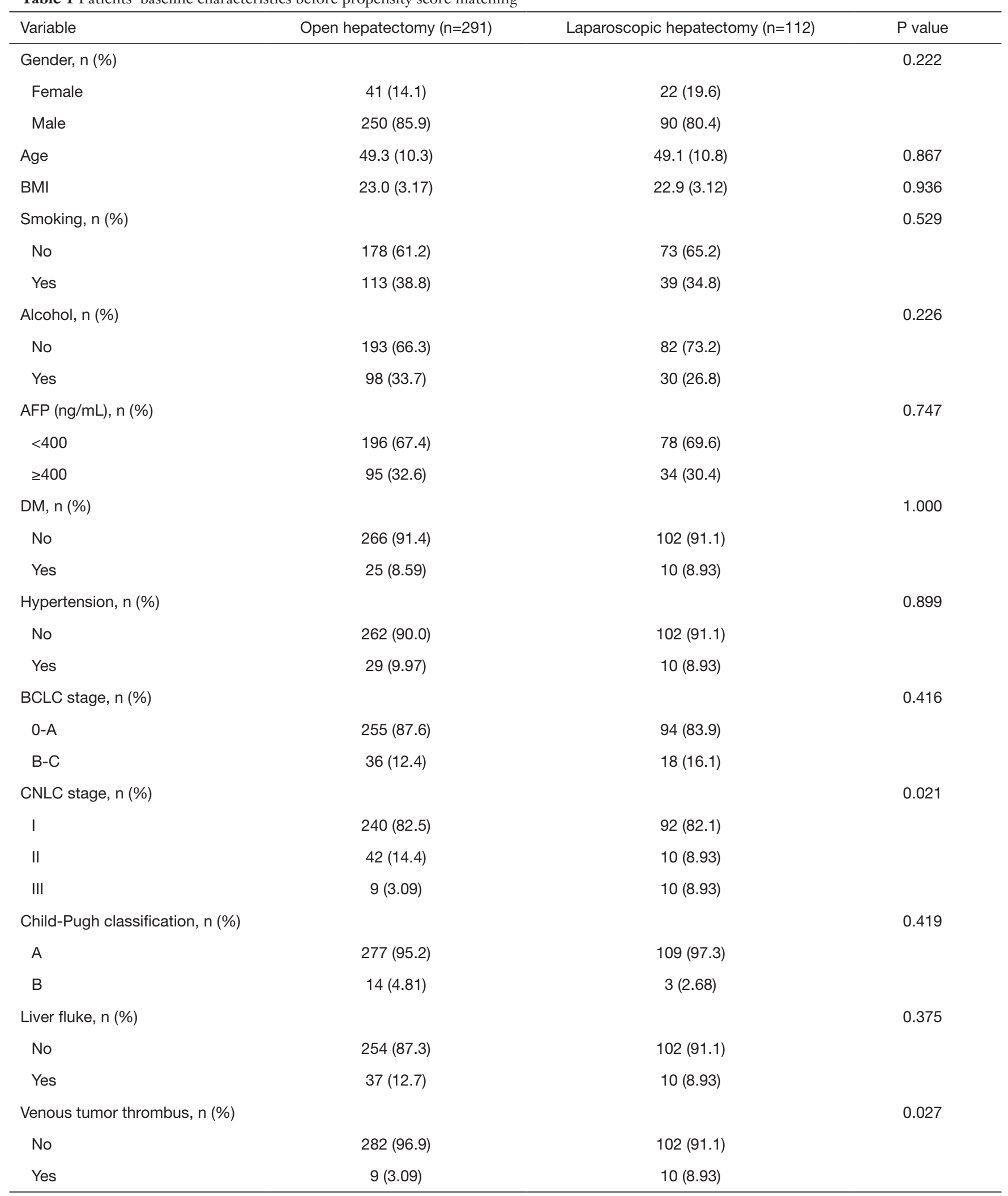

Table 1 (continued) 
Table 1 (continued)

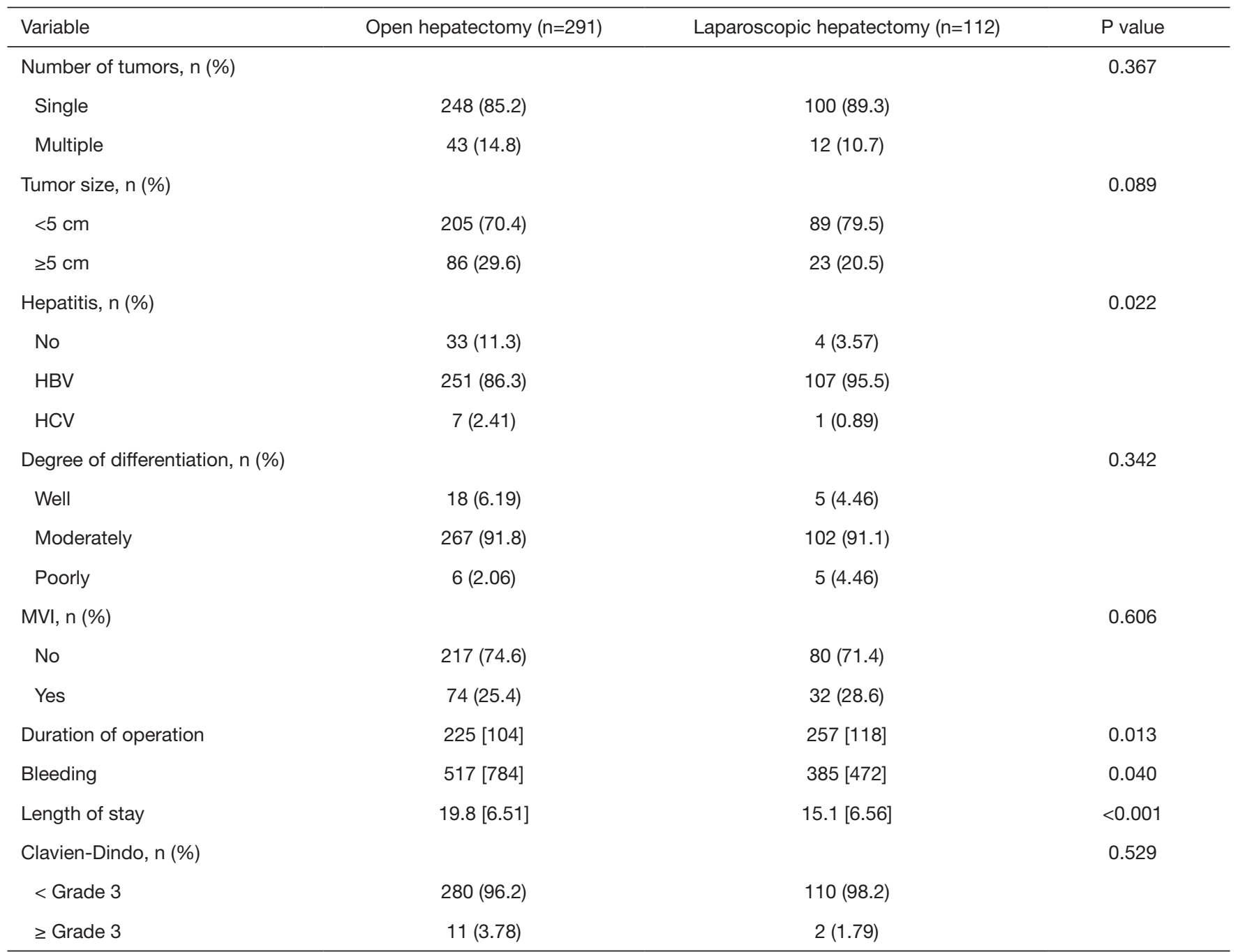

MVI, microvascular invasion; BMI, body mass index; AFP, $\alpha$-fetoprotein; DM, diabetes mellitus; BCLC, Barcelona clinic liver cancer staging; CNLC, China liver cancer staging.

thrombus $(\mathrm{P}=0.011)$, tumor size $\geq 5 \mathrm{~cm}(\mathrm{P}=0.001)$, MVI $(\mathrm{P}=0.001)$, and bleeding $(\mathrm{P}=0.012)$ were correlated with OS. BCLC stage $\mathrm{B}-\mathrm{C}(\mathrm{P}=0.031)$, CNLC stage II $(\mathrm{P}=0.014)$, multiple tumors $(\mathrm{P}=0.004)$, MVI $(\mathrm{P}=0.022)$, and bleeding $(\mathrm{P}=0.003)$ were correlated with RFS (Table 3). We selected clinically common prognostic factors for the multifactorial analysis, which indicated that venous tumor thrombus $(\mathrm{P}=0.049)$, tumor size $\geq 5 \mathrm{~cm}(\mathrm{P}=0.003)$, and MVI $(\mathrm{P}=0.032)$ were independent risk factors for OS. Multiple tumors $(\mathrm{P}=0.026)$ and bleeding $(\mathrm{P}=0.015)$ were independently correlated with RFS (Table 4). After PSM, univariate analysis showed that BCLC stage $\mathrm{B}-\mathrm{C}(\mathrm{P}=0.032)$, CNLC stage III ( $\mathrm{P}=0.018)$, Child-Pugh classification $\mathrm{B}(\mathrm{P}=0.013)$, venous tumor thrombus $(\mathrm{P}=0.017)$, tumor size $\geq 5 \mathrm{~cm}(\mathrm{P}=0.006)$, and MVI $(\mathrm{P}=0.026)$ were correlated with OS, while tumor size $\geq 5 \mathrm{~cm}(\mathrm{P}=0.033)$ and MVI $(\mathrm{P}=0.028)$ were correlated with RFS (Table 5).

\section{Survival analysis before and after PSM}

Before PSM, there was no significant difference in oncology outcomes between the two groups in OS $(\mathrm{P}=0.69)$ and RFS $(\mathrm{P}=0.25)$. The $\mathrm{OS}$ and RFS rates at 1,3 , and 5 years in $\mathrm{OH}$ were $95.7 \%, 81.4 \%, 67.7 \%, 87.4 \%, 68.9 \%$, and $54.9 \%$, respectively. The OS and RFS rates at 1,3 , and 5 years in LH group were $94.6 \%, 79.8 \%, 58 \%, 91.0 \%, 73.3 \%$, and 
Table 2 Patients' baseline characteristics after propensity score matching

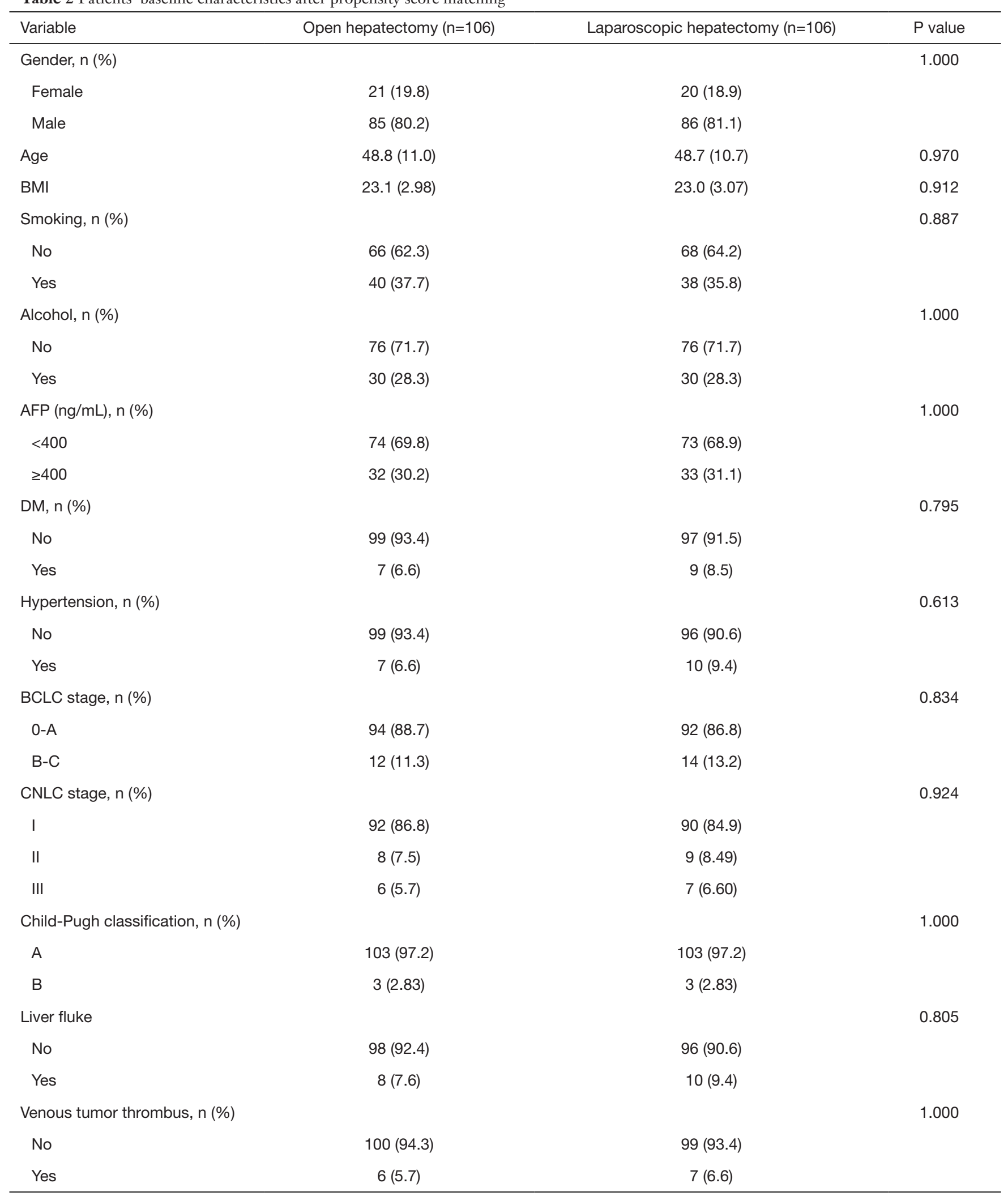

Table 2 (continued) 
Table 2 (continued)

\begin{tabular}{|c|c|c|c|}
\hline Variable & Open hepatectomy $(n=106)$ & Laparoscopic hepatectomy $(n=106)$ & $P$ value \\
\hline Single & $97(91.5)$ & $95(89.6)$ & \\
\hline Multiple & $9(8.5)$ & $11(10.4)$ & \\
\hline Tumor size, n (\%) & & & 0.728 \\
\hline$\geq 5 \mathrm{~cm}$ & $19(17.9)$ & $22(20.8)$ & \\
\hline Hepatitis, n (\%) & & & 0.873 \\
\hline No & $6(5.7)$ & $4(3.77)$ & \\
\hline HBV & $99(93.4)$ & $101(95.3)$ & \\
\hline Moderately & $98(92.4)$ & $97(91.5)$ & \\
\hline Poorly & $4(3.8)$ & $4(3.8)$ & \\
\hline MVI, n (\%) & & & 1.000 \\
\hline No & $76(71.7)$ & $75(70.8)$ & \\
\hline Yes & $30(28.3)$ & $31(29.2)$ & \\
\hline Duration of operation & $250[117]$ & 255 [116] & 0.734 \\
\hline Bleeding & 376 [340] & 389 [482] & 0.825 \\
\hline
\end{tabular}

AFP, $\alpha$-fetoprotein; DM, diabetes mellitus; BCLC, Barcelona clinic liver cancer; CNLC, China liver cancer; HBV, Hepatitis B virus; HCV, Hepatitis C virus; MVI, microvascular invasion.

46.4\%, respectively. After PSM, there was no significant difference in OS $(\mathrm{P}=0.7)$ and RFS $(\mathrm{P}=0.066)$ between the two groups. The rates of OS and RFS at 1,3 , and 5 years in $\mathrm{OH}$ were $93.2 \%, 80.2 \%, 72.4 \%, 83.5 \%, 65.3 \%$, and $41.7 \%$, respectively. The rates of OS and RFS at 1,3 , and 5 years in LH group were $94.3 \%, 79.9 \%, 57.5 \%, 90.5 \%, 74.1 \%$, and $53.0 \%$, respectively (Figure 3).

\section{Discussion}

Different stages of HCC lead to various treatment options, including hepatectomy, ablation, transarterial chemoembolization (TACE), targeted drug therapy, and liver transplantation $(15,16)$. For early HCC, hepatectomy is the best treatment. For unresectable tumors, neoadjuvant therapy and comprehensive treatment can be used to reduce the size of the tumor and classification stage so that surgical resection is possible. Hepatectomy as a radical resection can achieve a satisfactory outcome in oncology and can be used as a first-line treatment for early and intermediate HCC or as a pretransplant bridging therapy (17). The significance of translational therapy, which has emerged in recent years, is to provide patients with the opportunity for radical treatment, which may enable patients to achieve longer disease-free survival and OS. Advances in laparoscopic instruments and techniques have greatly improved 


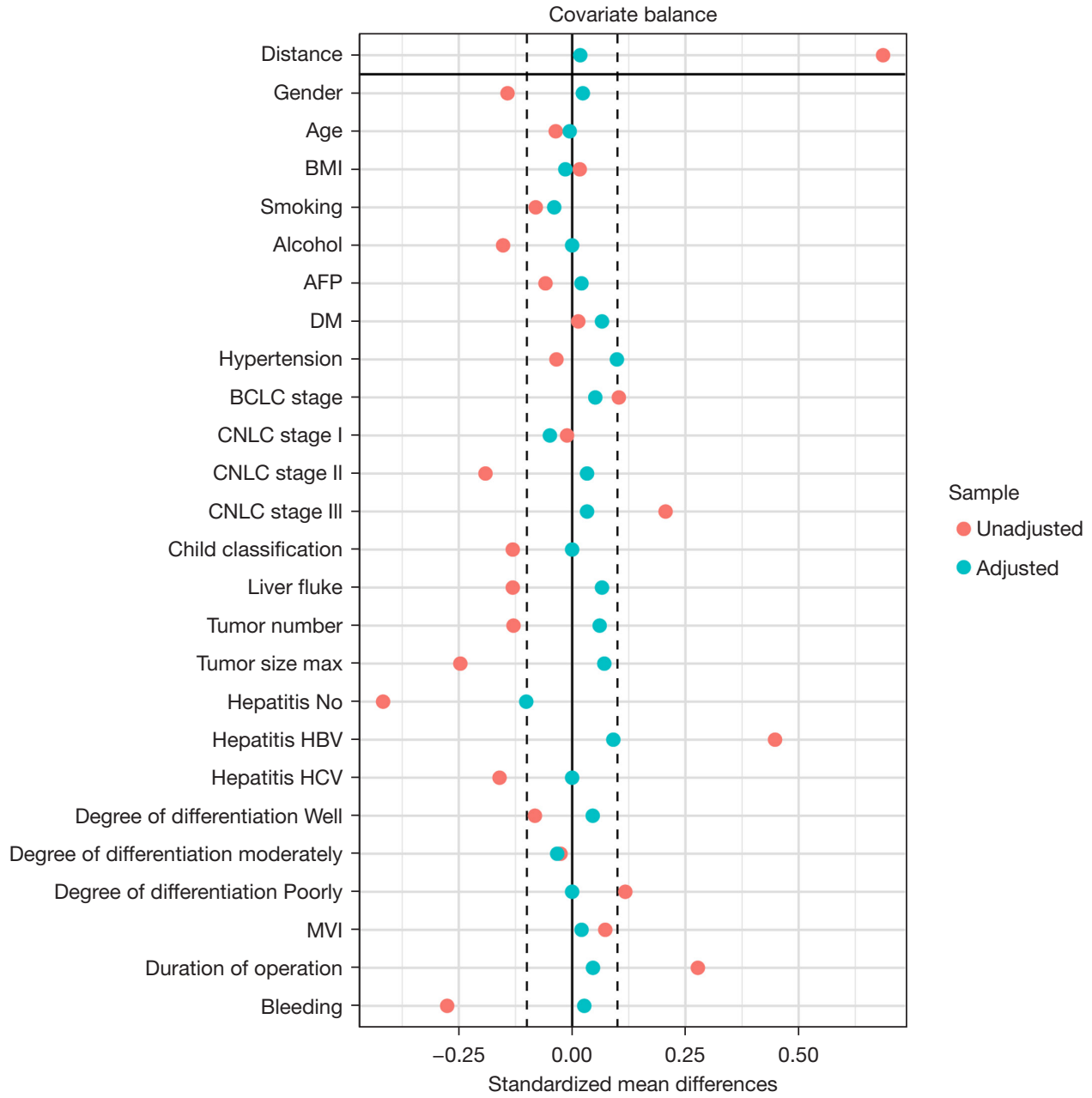

Figure 2 The balance test of propensity score matching. AFP, $\alpha$-fetoprotein; DM, diabetes mellitus; BCLC, Barcelona clinic liver cancer; CNLC, China liver cancer; HBV, Hepatitis B virus; HCV, Hepatitis C virus; MVI, microvascular invasion.

laparoscopic surgery and promoted the development of $\mathrm{LH}$, but the liver has a unique anatomical structure, and the procedure remains complex and challenging, especially for HCC patients with cirrhosis who need resection (18). Compared with $\mathrm{OH}, \mathrm{LH}$ has high-definition anatomical vision, and is also convenient for communication and learning among physicians. However, for tumors near large vessels or special parts, it is not convenient for laparoscopic instrument operation, and doctors with low experience need to master it after long-term practice.

The shortcomings of LH can be overcome to some extent by strengthening the training of surgical skills by senior or experienced physicians.

Clinical consensus is that cirrhosis is a high-risk factor for primary liver cancer, and the occurrence of cirrhosis can further shorten the survival of liver cancer patients (19). The degree of cirrhosis is closely related to the reserve function of the liver, especially the coagulation function. Patients with cirrhosis are prone to have complications, such as portal hypertension and impaired coagulation function. It is difficult to separate hardened liver tissue, and uncontrolled bleeding increases the risk of surgery in patients with cirrhosis (20). The majority of liver cancers in Guangxi are complicated by cirrhosis and require safe and effective surgical methods. The radical treatment of HCC may afford greater benefits for patients who have complex underlying liver diseases. With the widespread use of laparoscopy in general surgery, a large number of recent studies have reported the advantages of $\mathrm{LH}$, which can achieve complete tumor resection and tumor-free 
Table 3 Single analysis of OS and RFS before propensity score matching

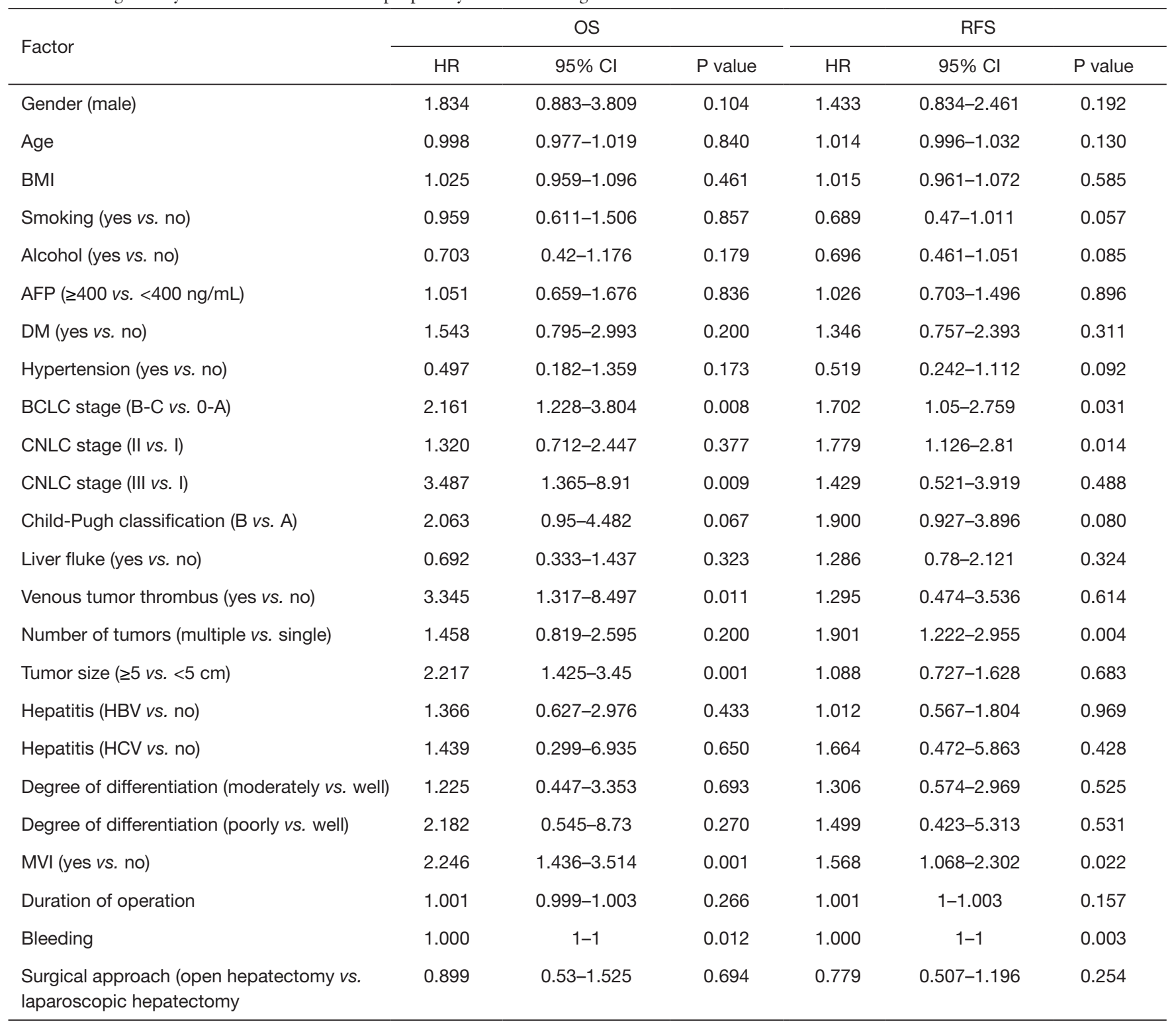

HR, hazard ratio; CI, confidence interval; OS, overall survival; RFS, relapse-free survival; AFP, $\alpha$-fetoprotein; DM, diabetes mellitus; BCLC, Barcelona clinic liver cancer; CNLC, China liver cancer; MVI, microvascular invasion.

resection margins (21). Accurate LH can reduce trauma and recurrence rates compared with early $\mathrm{OH}$ (22). In addition, laparoscopic robotic surgery, with its greater flexibility, can overcome the limitations of laparoscopic surgery alone and improve surgical accuracy. Furthermore, compared with open surgery, robotic surgery can shorten the length of hospital stay and reduce postoperative complications (23). However, prospective and randomized controlled studies comparing $\mathrm{LH}$ and $\mathrm{OH}$ have not been carried out.
This study retrospectively analyzed the prognosis of HCC patients with cirrhosis treated by laparoscopic and conventional $\mathrm{OH}$ from a single center.

Using PSM in this study, we found that tumor stage, Child-Pugh classification, venous tumor thrombus, tumor size $\geq 5 \mathrm{~cm}$, and MVI were independent risk factors affecting postoperative OS in HCC patients. Tumor size $\geq 5 \mathrm{~cm}$ and MVI were also correlated with RFS. It has been reported that Child-Pugh classification is associated with 
Table 4 Univariate analysis of OS and RFS before propensity score matching

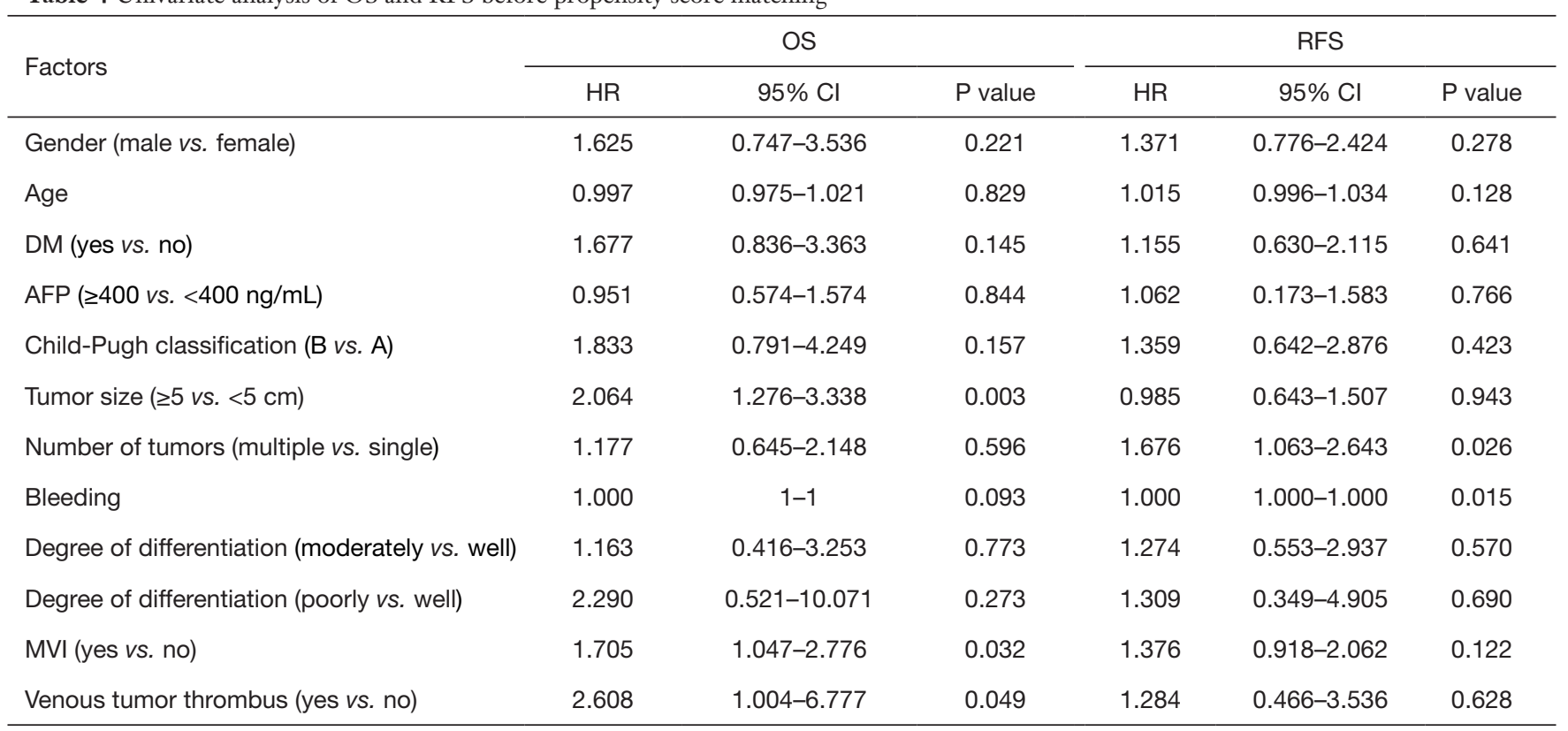

OS, overall survival; RFS, relapse-free survival; DM, diabetes mellitus; AFP, $\alpha$-fetoprotein; MVI, microvascular invasion.

hepatectomy (Child-Pugh B score) and a high incidence of postoperative complications in advanced cirrhotic HCC (24). The mortality rate of hepatectomy in ChildPugh grade A, B, and C cirrhosis patients was $10 \%, 17 \%$, and $63 \%$, respectively (25). Portal vein cancer thrombus can easily form intrahepatic metastasis, and some researchers claim it should be listed as a contraindication for surgery (26). However, some scholars believe that in cases with good liver function and comprehensive preoperative planning and evaluation, HCC complicated by portal vein cancer thrombus or portal hypertension can also be treated with surgery, and some patients can still benefit from surgical resection. Results have also shown that long-term postoperative survival was better than other treatments (27-30). MVI is common in HCC and has been repeatedly shown to be a poor long-term prognostic factor associated with early tumor recurrence and reduced survival (31-33). Studies have identified tumor size $(>2-3 \mathrm{~cm})$, number of tumors, and MVI as risk factors for HCC recurrence $(34,35)$. MVI is an important risk factor for early postoperative recurrence, and there are significant differences in the early RFS rates of patients with or without MVI or mild versus severe MVI. Even for patients with small HCC, MVI will increase the recurrence rate of HCC, which is not conducive to the long-term survival of patients (36-40). Our results are similar to those previously reported, which attests to the reliability of the current study analysis.

Long-term oncology prognosis and disease-free survival are critical in determining the role of LH in HCC management. Most of the current reports on long-term prognosis show no significant differences between LH and $\mathrm{OH}$ surgical procedures and 5-year OS and RFS, even in patients with cirrhosis (41-44). However, a previous metaanalysis compared the results of $\mathrm{LH}$ and $\mathrm{OH}$ in liver cancer patients with cirrhosis and found that the 5 -year OS of LH was better than that of $\mathrm{OH}$ (45), which may be related to the number of studies included and the lack of longterm prognostic data. In our study, there was no significant difference between the OS and RFS of patients in the LH and $\mathrm{OH}$ groups, whether before or after PSM. Similar to most results from recent studies, it also reflects the reliability of the oncology outcome analysis in our study. Therefore, we suggest that $\mathrm{LH}$ can be used to shorten the operation time for tumors located in the left half of the liver or superficial. For patients with special tumor location or portal hypertension, $\mathrm{OH}$ can be used to improve surgical safety. Our study focused on patient outcomes, so hospitalization costs were not explored in depth.

The retrospective nature of the current study contains inherent flaws due to the lack of randomization, the small sample size, and the single-center design, which may have affected the strength and validity of our results. In addition, 
Table 5 Single-factor analysis of OS and RFS after propensity score matching

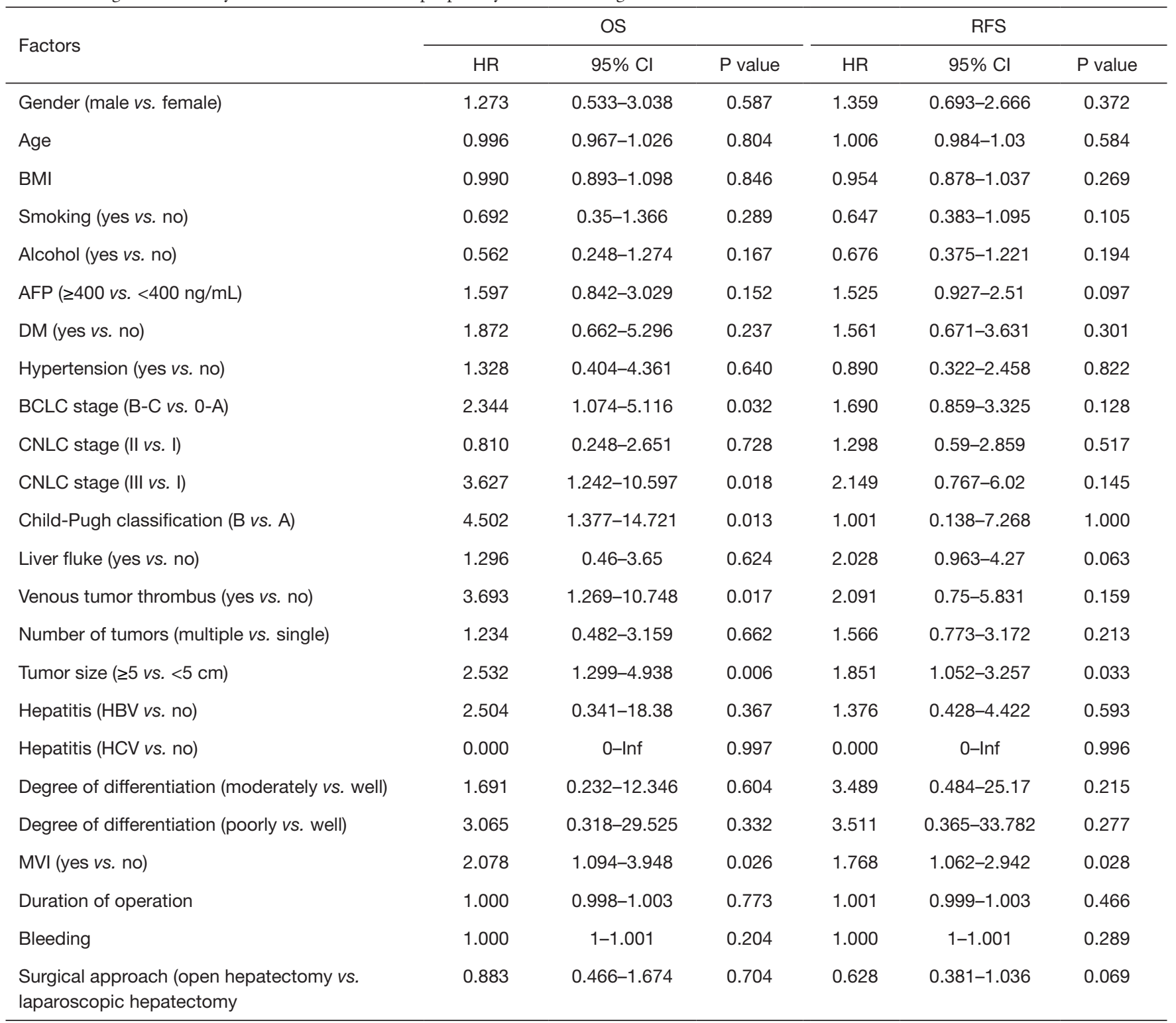

OS, overall survival; RFS, relapse-free survival; AFP, $\alpha$-fetoprotein; DM, diabetes mellitus; BCLC, Barcelona clinic liver cancer; CNLC, China liver cancer; MVI, microvascular invasion. 
A
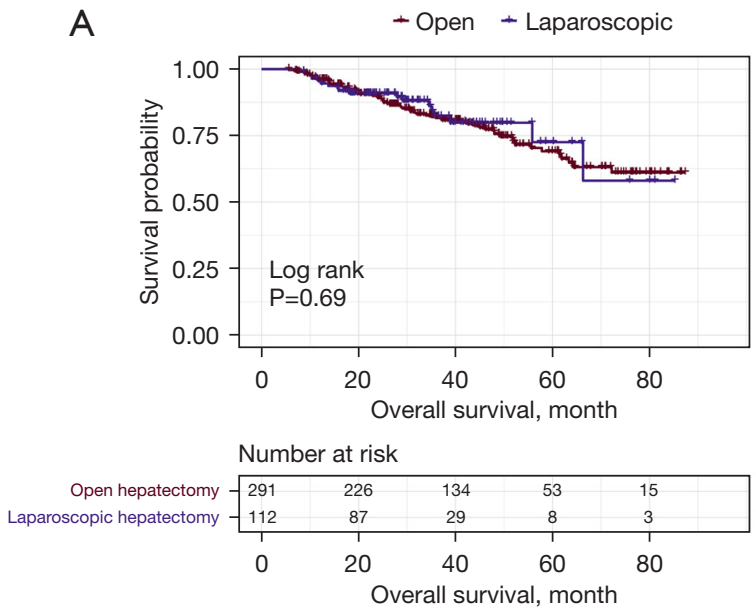

C
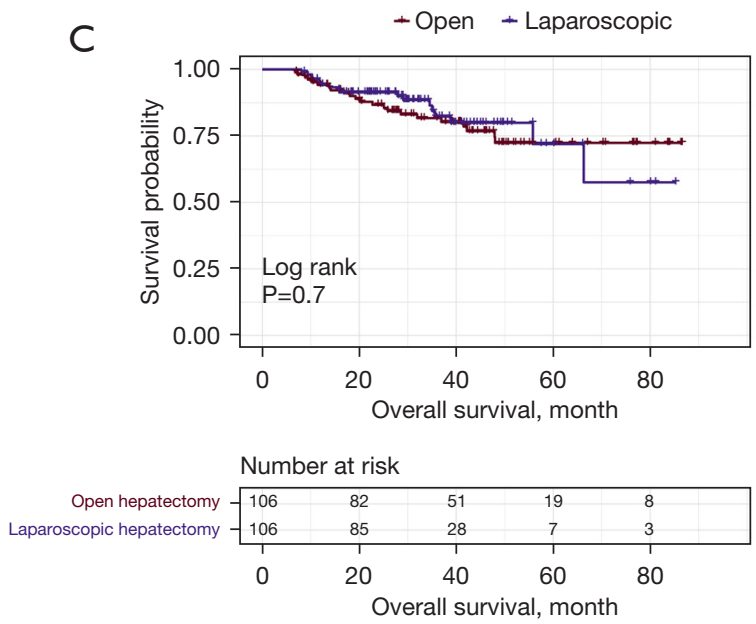

B
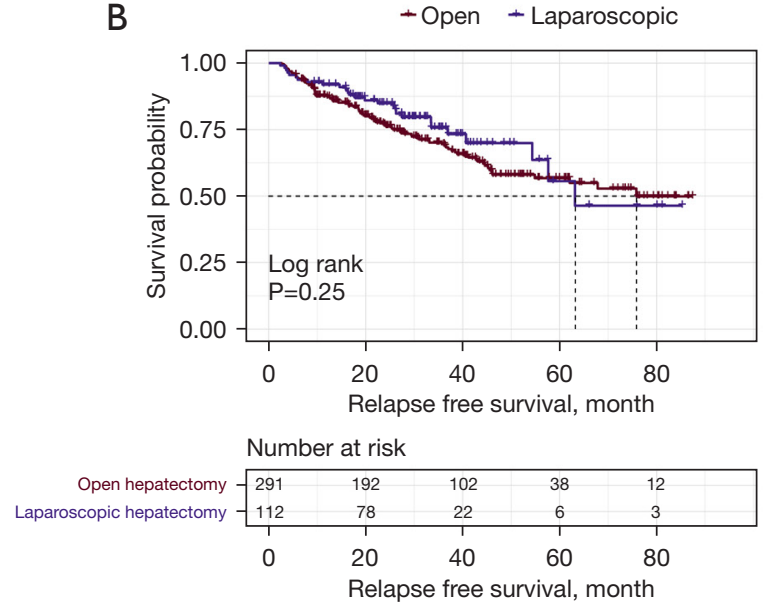

D
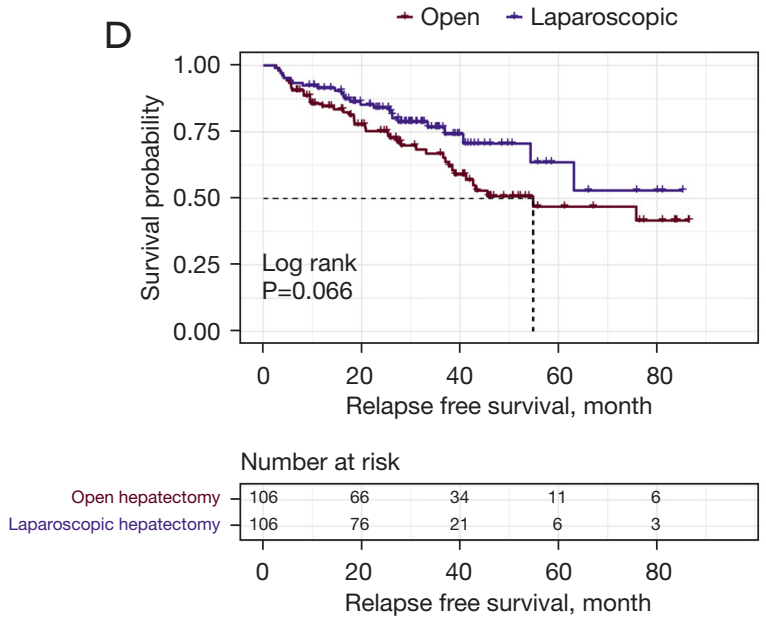

Figure 3 Analysis of the survival curve before and after PSM. (A,B) OS and RFS before PSM; (C,D) OS and RFS after PSM. PSM, propensity score matching; OS, overall survival; RFS, relapse-free survival.

the primary etiology of HCC in our region is the hepatitis $B$ virus infection, which differs from that in European and American countries. Bias matching can minimize selection bias and the effect of potential covariates, but randomized controlled trials are still needed to investigate short - and long-term patient outcomes.

\section{Conclusions}

In conclusion, our study showed that LH had a similar postoperative OS and RFS compared with traditional open surgery for HCC patients with cirrhosis. LH can be used as a feasible treatment option for HCC patients with cirrhosis.

\section{Acknowledgments}

Funding: The National Natural Science Foundation of China (Grant No. 81560535, 81802874, 81072321) supported the data collection and analysis, and the Natural Science Foundation of the Guangxi Province of China (Grant No. 2018 GXNSFBA138013) and Guangxi Key R\&D Program (Grant No. GKEAB18221019) supported the manuscript writing.

\section{Footnote}

Reporting Checklist: The authors have completed the 
STROBE reporting checklist. Available at https://dx.doi. org/10.21037/atm-21-5833

Data Sharing Statement: Available at https://dx.doi. org/10.21037/atm-21-5833

Conflicts of Interest: All authors have completed the ICMJE uniform disclosure form (available at https://dx.doi. org/10.21037/atm-21-5833). The authors have no conflicts of interest to declare.

Ethical Statement: The authors are accountable for all aspects of the work in ensuring that questions related to the accuracy or integrity of any part of the work are appropriately investigated and resolved. The study was conducted in accordance with the Declaration of Helsinki (as revised in 2013). This study was approved by the Ethics Committee of the First Affiliated Hospital of Guangxi Medical University (No. 2021-KY-E-277). Individual consent for this retrospective analysis was waived.

Open Access Statement: This is an Open Access article distributed in accordance with the Creative Commons Attribution-NonCommercial-NoDerivs 4.0 International License (CC BY-NC-ND 4.0), which permits the noncommercial replication and distribution of the article with the strict proviso that no changes or edits are made and the original work is properly cited (including links to both the formal publication through the relevant DOI and the license). See: https://creativecommons.org/licenses/by-nc-nd/4.0/.

\section{References}

1. Chen $\mathrm{W}$, Zheng R, Baade PD, et al. Cancer statistics in China, 2015. CA Cancer J Clin 2016;66:115-32.

2. Xie DY, Ren ZG, Zhou J, et al. 2019 Chinese clinical guidelines for the management of hepatocellular carcinoma: updates and insights. Hepatobiliary Surg Nutr 2020;9:452-63.

3. Sperber AD, Bangdiwala SI, Drossman DA, et al. Worldwide Prevalence and Burden of Functional Gastrointestinal Disorders, Results of Rome Foundation Global Study. Gastroenterology 2021;160:99-114.e3.

4. Kanazawa A, Tsukamoto T, Shimizu S, et al. Impact of laparoscopic liver resection for hepatocellular carcinoma with F4-liver cirrhosis. Surg Endosc 2013;27:2592-7.

5. Reich H, McGlynn F, DeCaprio J, et al. Laparoscopic excision of benign liver lesions. Obstet Gynecol
1991;78:956-8.

6. Fong Y, Sun RL, Jarnagin W, et al. An analysis of 412 cases of hepatocellular carcinoma at a Western center. Ann Surg 1999;229:790-9; discussion 799-800.

7. Vanounou T, Steel JL, Nguyen KT, et al. Comparing the clinical and economic impact of laparoscopic versus open liver resection. Ann Surg Oncol 2010;17:998-1009.

8. Kaneko H, Takagi S, Otsuka Y, et al. Laparoscopic liver resection of hepatocellular carcinoma. Am J Surg 2005;189:190-4.

9. Ramirez PT, Frumovitz M, Pareja R, et al. Minimally Invasive versus Abdominal Radical Hysterectomy for Cervical Cancer. N Engl J Med 2018;379:1895-904.

10. Jiang X, Liu L, Zhang Q, et al. Laparoscopic versus open hepatectomy for hepatocellular carcinoma: long-term outcomes. J BUON 2016;21:135-41.

11. Belli G, Limongelli P, Fantini C, et al. Laparoscopic and open treatment of hepatocellular carcinoma in patients with cirrhosis. Br J Surg 2009;96:1041-8.

12. Yamashita Y, Ikeda T, Kurihara T, et al. Long-term favorable surgical results of laparoscopic hepatic resection for hepatocellular carcinoma in patients with cirrhosis: a single-center experience over a 10-year period. J Am Coll Surg 2014;219:1117-23.

13. Department of Medical Administration, National Health and Health Commission of the People's Republic of China. Guidelines for diagnosis and treatment of primary liver cancer in China (2019 edition). Zhonghua Gan Zang Bing Za Zhi 2020;28:112-28.

14. Dindo D, Demartines N, Clavien PA. Classification of surgical complications: a new proposal with evaluation in a cohort of 6336 patients and results of a survey. Ann Surg 2004;240:205-13.

15. Forner A, Reig M, Bruix J. Hepatocellular carcinoma. Lancet 2018;391:1301-14.

16. Xie X, Yu Y. Prognosis value of liver stiffness measurements by 2D-SWE in primary HBV-positive hepatocellular carcinoma following radiofrequency ablation. Transl Cancer Res 2020;9:2518-26.

17. Bruix J, Sherman M, Llovet JM, et al. Clinical management of hepatocellular carcinoma. Conclusions of the Barcelona-2000 EASL conference. European Association for the Study of the Liver. J Hepatol 2001;35:421-30.

18. Komatsu S, Brustia R, Goumard C, et al. Laparoscopic versus open major hepatectomy for hepatocellular carcinoma: a matched pair analysis. Surg Endosc 2016;30:1965-74.

19. Nilsson E, Anderson H, Sargenti K, et al. Risk and 
outcome of hepatocellular carcinoma in liver cirrhosis in Southern Sweden: a population-based study. Scand J Gastroenterol 2019;54:1027-32.

20. Worhunsky DJ, Dua MM, Tran TB, et al. Laparoscopic hepatectomy in cirrhotics: safe if you adjust technique. Surg Endosc 2016;30:4307-14.

21. Cherian PT, Mishra AK, Kumar P, et al. Laparoscopic liver resection: wedge resections to living donor hepatectomy, are we heading in the right direction? World J Gastroenterol 2014;20:13369-81.

22. Luo F, Zhou J, Yan W. A comparative study of laparoscopic precise hepatectomy with conventional open hepatectomy in the treatment of primary hepatocellular cancer. J BUON 2019;24:1943-9.

23. Yang HY, Rho SY, Han DH, et al. Robotic major liver resections: Surgical outcomes compared with open major liver resections. Ann Hepatobiliary Pancreat Surg 2021;25:8-17.

24. Berardi G, Morise Z, Sposito C, et al. Development of a nomogram to predict outcome after liver resection for hepatocellular carcinoma in Child-Pugh B cirrhosis. J Hepatol 2020;72:75-84.

25. Neeff H, Mariaskin D, Spangenberg HC, et al. Perioperative mortality after non-hepatic general surgery in patients with liver cirrhosis: an analysis of 138 operations in the 2000s using Child and MELD scores. J Gastrointest Surg 2011;15:1-11.

26. European Association For The Study Of The Liver; European Organisation For Research And Treatment Of Cancer. EASL-EORTC clinical practice guidelines: management of hepatocellular carcinoma. J Hepatol 2012;56:908-43.

27. Chen X, Zhai J, Cai X, et al. Severity of portal hypertension and prediction of postoperative liver failure after liver resection in patients with Child-Pugh grade A cirrhosis. Br J Surg 2012;99:1701-10.

28. Liu PH, Lee YH, Hsia CY, et al. Surgical resection versus transarterial chemoembolization for hepatocellular carcinoma with portal vein tumor thrombosis: a propensity score analysis. Ann Surg Oncol 2014;21:1825-33.

29. Kokudo T, Hasegawa K, Matsuyama Y, et al. Survival benefit of liver resection for hepatocellular carcinoma associated with portal vein invasion. J Hepatol 2016;65:938-43.

30. Wang K, Guo WX, Chen MS, et al. Multimodality Treatment for Hepatocellular Carcinoma With Portal Vein Tumor Thrombus: A Large-Scale, Multicenter, Propensity Mathching Score Analysis. Medicine (Baltimore) 2016;95:e3015.

31. Cong WM, Bu H, Chen J, et al. Practice guidelines for the pathological diagnosis of primary liver cancer: 2015 update. World J Gastroenterol 2016;22:9279-87.

32. Lei Z, Li J, Wu D, et al. Nomogram for Preoperative Estimation of Microvascular Invasion Risk in Hepatitis B Virus-Related Hepatocellular Carcinoma Within the Milan Criteria. JAMA Surg 2016;151:356-63.

33. Huang C, Zhu X, Ji Y, et al. Microvascular invasion has limited clinical values in hepatocellular carcinoma patients at Barcelona Clinic Liver Cancer (BCLC) stages 0 or B. BMC cancer 2017;17:58.

34. Shindoh J, Hasegawa K, Inoue Y, et al. Risk factors of post-operative recurrence and adequate surgical approach to improve long-term outcomes of hepatocellular carcinoma. HPB (Oxford) 2013;15:31-9.

35. Colecchia A, Schiumerini R, Cucchetti A, et al. Prognostic factors for hepatocellular carcinoma recurrence. World J Gastroenterol 2014;20:5935-50.

36. Shah SA, Greig PD, Gallinger S, et al. Factors associated with early recurrence after resection for hepatocellular carcinoma and outcomes. J Am Coll Surg 2006;202:275-83.

37. Sumie S, Kuromatsu R, Okuda K, et al. Microvascular invasion in patients with hepatocellular carcinoma and its predictable clinicopathological factors. Ann Surg Oncol 2008;15:1375-82.

38. Fan LF, Zhao WC, Yang N, et al. Alpha-fetoprotein: the predictor of microvascular invasion in solitary small hepatocellular carcinoma and criterion for anatomic or non-anatomic hepatic resection. Hepatogastroenterology 2013;60:825-36.

39. Du M, Chen L, Zhao J, et al. Microvascular invasion (MVI) is a poorer prognostic predictor for small hepatocellular carcinoma. BMC Cancer 2014;14:38.

40. Ünal E, İdilman İS, Akata D, et al. Microvascular invasion in hepatocellular carcinoma. Diagn Interv Radiol 2016;22:125-32.

41. Sarpel U, Hefti MM, Wisnievsky JP, et al. Outcome for patients treated with laparoscopic versus open resection of hepatocellular carcinoma: case-matched analysis. Ann Surg Oncol 2009;16:1572-7.

42. Aldrighetti L, Guzzetti E, Pulitanò C, et al. Case-matched analysis of totally laparoscopic versus open liver resection for HCC: short and middle term results. J Surg Oncol 2010;102:82-6.

43. Parks KR, Kuo YH, Davis JM, et al. Laparoscopic versus open liver resection: a meta-analysis of long-term outcome. HPB (Oxford) 2014;16:109-18. 
44. Chang SK, Tay CW, Shen L, et al. Long-Term

Oncological Safety of Minimally Invasive Hepatectomy in Patients with Hepatocellular Carcinoma: A Case-Control Study. Ann Acad Med Singap 2016;45:91-7.

45. Chen J, Bai T, Zhang Y, et al. The safety and efficacy of

Cite this article as: He Y, Fang D, Liang T, Mo S, Meng Y, Chen Z, Zhao S, Liao Y, Huang K, Nong S, Zhou W, Han C, Peng T. Laparoscopic versus open hepatectomy for hepatocellular carcinoma with cirrhosis: a single-center propensity score matching analysis. Ann Transl Med 2021;9(23):1733. doi: 10.21037/atm-21-5833 laparoscopic and open hepatectomy in hepatocellular carcinoma patients with liver cirrhosis: a systematic review. Int J Clin Exp Med 2015;8:20679-89.

(English Language Editor: D. Fitzgerald) 